\title{
Concentric Mosaic(s), Planar Motion and 1D Cameras
}

\author{
Long QUAN ${ }^{1} \quad$ Le LU $^{3,2} \quad$ Heung-Yeung SHUM ${ }^{2} \quad$ Maxime LHUILLIER ${ }^{1}$ \\ ${ }^{1}$ CNRS-INRIA, 655 avenue de l'Europe, 38330 Montbonnot, France \\ ${ }^{2}$ Microsoft Research China, Beijing 100080, P.R. China \\ ${ }^{3}$ National Lab of Pattern Recognition, Chinese Academy of Sciences, Beijing 100080, P.R. China
}

\begin{abstract}
General SFM methods give poor results for images captured by constrained motions such as planar motion of concentric mosaics $(C M)$. In this paper, we propose new SFM algorithms for both images captured by CM and composite mosaic images from CM. We first introduce $1 D$ affine camera model for completing $1 D$ camera models. Then we show that a $2 D$ image captured by $C M$ can be decoupled into two $1 D$ images: one $1 D$ projective and one $1 D$ affine; a composite mosaic image can by rebinned into a calibrated $1 D$ panorama projective camera. Finally we describe subspace reconstruction methods and demonstrate both in theory and experiments the advantage of the decomposition method over the general SFM methods by incorporating the constrained motion into the earliest stage of motion analysis.
\end{abstract}

Key words: SFM, planar motion, 1D camera, vision geometry, image-based rendering.

\section{Introduction}

Image-Based Rendering and Concentric mosaics Recently there has been much interest in computer vision and graphics in image-based rendering methods [16]. They generate new views of scenes from novel viewpoints, using a collection of images as the underlying scene representation. When the sampling rate is sparse, this is very similar to classical computer vision based $3 \mathrm{D}$ reconstruction, either explicit $[18,21,13]$ or implicit $[14,5]$ with texture mapping. When sampling is dense, a large amount of work $[16,15,11]$ has been developed based on plenoptic functions. This models all sets of rays seen from all points, considering each image as a set of rays. The major challenge is the very high dimensionality of such plenoptic functions. Many simplifying assumptions that limit the underlying viewing space have been introduced: 5D plenoptic modeling [16], 4D Lightfield/Lumigraph [15, 11], 3D concentric mosaics [23] and 2D panorama [19, 7, 25]. Among all these approaches, concentric mosaics [23] is a good trade-off between the ease of acquisition and viewing space. The camera motion of CM is constrained to planar concentric circle, CMs are created by composing slit images taken at different locations along each circle. CMs index all imput image rays naturally in 3 parameters: radius, rotation angle and vertical elevation.

As concentric mosaics are rendered by ray interpolation, like Lightfield, based on constant depth without geometric correction, it is necessary to compute the geometric structure to be able to handle more complex than constantdepth-type scenes and correct the inherent vertical distortion [23].

Planar motion A camera moving in a plane and rotating about an axis perpendicular to that plane is called planar motion. This is the typical motion undergone by a vehicle moving on a plane. It has been shown in $[6,29]$ that an affine reconstruction is possible provided the constant internal parameters of the camera. A more complete self-calibration method with constant internal parameters from planar motion has been proposed in [1,2].

1D camera A 1D projective camera maps a point in $\mathcal{P}^{2}$ (the projective space of dimension 2) onto a point in $\mathcal{P}^{1}$ with a $2 \times 3$ matrix, by analogy to a $2 \mathrm{D}$ projective camera that maps a point in $\mathcal{P}^{3}$ to a point in $\mathcal{P}^{2}$. Many work has been done for both uncalibrated and calibrated $1 \mathrm{D}$ projective cameras $[4,20]$. What is more interesting is that the usual 2D camera model could be related to this 1D camera model in [20] for affine camera case and in [9] for planar motion case.

The first observation is that CM capture device undergoes a perfect planar motion. The geometry of CMs inherits that of planar motion.

For a complete decomposition of concentric mosaic images, we introduce a new 1D affine camera model.

We first show that a image captured by CM can be decoupled into two 1D cameras, one horizontal 1D projective camera and one vertical 1D affine camera. The scene depth can be recovered by the horizontal 1D projective camera while the height from the vertical 1D affine camera. Then we show that a composite mosaic image can be rebinned into a calibrated 1D panorama projective camera. The unifying thema is that the geometry of concentric mo- 
camera and study its geometric properties. Finally we describe subspace reconstruction methods and demonstrate both in theory and experiments the advantage of the decomposition method over the general SFM algorithms by integrating the prior motion constraints into the estimation procedure.

The paper is organised as follows. Section 2 describes the geometric analysis of images captured by $\mathrm{CM}$ and Section 2 the geometric analysis of composite mosaic images. Then, we describe 1D affine camera model and 2D reconstruction from 1D images in Section 4. Experimental results are given in Section 5. Finally some concluding remarks are given in Section 6. Throughout the paper, vectors are denoted in lower case boldface, matrices and tensors in upper case boldface. Scalars are any plain letters or lower case Greek.

\section{Geometry of images captured by CMs}

The camera mounted on the CM capture system rotates around the fixed vertical axis. The original images (before mosaic composition) of concentric mosaics are therefore constrained by a planar motion. A general approach described in $[29,6,1,28]$ consists of first computing a projective structure, then extracting fixed entities by planar motion or by constant internal calibration. We follow a different approach [9] in which 2D image of a planar motion is reduced to the trifocal line image. We take this decomposition principle futher by introducing a complete decoupling of the 2D image into two complementary 1D images, one on the trifocal (motion) line and the other on the pencil of epipolar lines. The 3D space is accordingly decomposed into two orthogonal subspaces, one of dimension 2 represented by the trifocal plane and another of dimension 1 by the pencil of epipolar planes.

The 1D cameras defined this way are virtual and almost no physical points live in these subspaces. The virtual points on the trifocal plane could be simply created by projecting the 2D image points onto the trifocal line [9]. The second 1D camera is imaging the pencil of epipolar planes in space. It is in fact a sort of dual 1D imaging as the ambient space elements are now dual elements, i.e. planes. Even more, this dual space is only of dimension 1 , so the projection is described by a $2 \times 21 \mathrm{D}$ homography from $\mathcal{P}^{1}$ to $\mathcal{P}^{1}$ instead of a $2 \times 3$ matrix from $\mathcal{P}^{2}$ to $\mathcal{P}^{1}$. This is similar to $2 \mathrm{D}$ homography description of planar scenes by 2D cameras. Any image line intersecting the epipolar pencil produces a 1D projective image of the epipolar pencils. This decoupling of 2D image into 1D images is only possible provided that the trifocal tensor or fundamental matrices have been estimated, this make the practical implementation of the decomposition more sensitive to the prior geometric computation.

Now applying this decomposition to the images of the tical. The vanishing point of the rotation axes is therefore the point at infinity of the vertical direction. This simplifies the projection of 2D image points onto the trifocal line: the horizontal $u$-coordinates of points makes up the $1 \mathrm{D}$ image line. With the vanishing point of rotation axes at vertical infinity, the second $1 \mathrm{D}$ projection also gets simplified as the vertical 1D homography now becomes an affine transformation. The vertical 1D camera is therefore a kind of affine camera instead of projective. This motivates the definition and analysis of 1D affine camera in Section 4.1.

Now let introduce the space Euclidean coordinate frame such that $x z$ plane is the ground plane and the $y$ axis is normal to the ground plane. The horizontal and vertical pixel coordinates are $u$ and $v$. The $2 \mathrm{D}$ camera matrix $\mathbf{P}$ relates them by $\lambda \mathbf{u}=\mathbf{P x}$. The constrained motion preserves the point at infinity in $y$ axis direction and the ground plane $y=0$ is imaged into the horizon line $v=0$. The camera moving this way has the matrix of the form [29]:

$$
\left(\begin{array}{llll}
a & 0 & b & c \\
0 & d & 0 & 0 \\
e & 0 & f & g
\end{array}\right)
$$

Projecting 3D points with coordinates $(x, y, z, t)^{T}$ into two orthogonal subspaces of dimension 2 with coordinates $(x, 0, z, t)^{T}$ and of dimension 1 with coordinates $(0, y, 0, t)^{T}$. Working with points in these subspaces gives

$$
\lambda\left(\begin{array}{l}
u \\
1
\end{array}\right)=\left(\begin{array}{lll}
a & b & c \\
e & f & g
\end{array}\right)\left(\begin{array}{l}
x \\
z \\
t
\end{array}\right),
$$

which is a $1 \mathrm{D}$ projective camera from $\mathcal{P}^{2}$ to $\mathcal{P}^{1}$; and

$$
\mu\left(\begin{array}{l}
v \\
1
\end{array}\right)=\left(\begin{array}{ll}
d & 0 \\
0 & g
\end{array}\right)\left(\begin{array}{l}
y \\
t
\end{array}\right) .
$$

which is a $1 \mathrm{D}$ affine camera from $\mathcal{P}^{2}$ to $\mathcal{P}^{1}$.

Whence these subspaces have been reconstructed, the 3D space point can be recovered by linearly combining $\lambda(x, 0, z, 1)^{T}+\mu(0,1,0,0)^{T}$ with proper scales $\lambda / \mu$ fixed by $y / t$.

Alternatively, this decoupling schema may be viewed as two 1D cameras directly from 3D spaces by $\left(\begin{array}{llll}a & 0 & b & c \\ e & 0 & f & g\end{array}\right)$ and $\left(\begin{array}{llll}0 & d & 0 & 0 \\ 0 & 0 & 0 & g\end{array}\right)$, but these 3D to 1D cameras being singular do not bring new insight.

In summary, this gives a simplified SFM algorithm for images captured by CMs.

- decoupling the original 2D images into two 1D images by $(u, v)^{T} \mapsto u$ and $(u, v)^{T} \mapsto v$;

- reconstructing 2D points $(x, z, t)$ and 1D points $(y, t)$

- reconstructing $3 \mathrm{D}$ points by spanning the subspaces $t(x, 0, z, 1)^{T}+y(0,1,0,0)^{T}$. 


$$
\left(\begin{array}{ccc}
1 & 0 & p_{23}
\end{array}\right)=\left(\begin{array}{ll}
\mathbf{0}_{1 \times 2} & \mathbf{t}_{2 \times 1}
\end{array}\right)
$$

It maps the finite points $(x, y, 1)^{T}$ onto finite image points $(u, 1)^{T}$ with

$$
u=\mathbf{m}_{1 \times 2}\left(\begin{array}{l}
x \\
y
\end{array}\right)+t .
$$

If we further use relative cooridinates with respect to a given reference point, for instance, the centroid of the point set, $\Delta u=u-u_{r}$ in $\mathbb{R}^{1}$ and $(\Delta x, \Delta y)^{T}=$ $\left(x-x_{r}, y-y_{r}\right)^{T}$ in $\mathbb{R}^{2}$. The translational component $t$ is cancelled and the projection for finite points in relative coordinates are therefore:

$$
\Delta u=\mathbf{m}_{1 \times 2}\left(\begin{array}{c}
\Delta x \\
\Delta y
\end{array}\right) .
$$

This is the basic 1D affine camera projection. We now examine the geometric constraints available for points seen in multiple views similar to the $2 \mathrm{D}$ camera case $[22,24,12,27,8]$.

Let three views of the point $\Delta \mathbf{x}$ be given as follows:

$$
\left\{\begin{aligned}
\Delta u & =\Delta \mathbf{m} \mathbf{x} \\
\Delta u^{\prime} & =\Delta \mathbf{m}^{\prime} \mathbf{x} \\
\Delta u^{\prime \prime} & =\Delta \mathbf{m}^{\prime \prime} \mathbf{x}
\end{aligned}\right.
$$

These can be rewritten in matrix form as

$$
\left(\begin{array}{cc}
\mathbf{m}^{T} & \Delta u \\
\mathbf{m}^{\prime T} & \Delta u^{\prime} \\
\mathbf{m}^{\prime \prime T} & \Delta u^{\prime \prime}
\end{array}\right)\left(\begin{array}{c}
\Delta \mathbf{x} \\
-\lambda
\end{array}\right)=\mathbf{0}
$$

The vector $(\Delta \mathbf{x},-\lambda)^{T}$ cannot be zero, so

$$
\left|\begin{array}{cc}
\mathbf{m}^{T} & \Delta u \\
\mathbf{m}^{\prime T} & \Delta u^{\prime} \\
\mathbf{m}^{\prime \prime} & \Delta u^{\prime \prime}
\end{array}\right|=\left|\begin{array}{ccc} 
& & \Delta u \\
\mathbf{a} & \mathbf{b} & \Delta u^{\prime} \\
\Delta u^{\prime \prime}
\end{array}\right|=0 .
$$

The expansion of this determinant produces a linear constraint for the three 1D affine views

$$
(\mathbf{a} \times \mathbf{b})^{T}\left(\begin{array}{c}
\Delta u \\
\Delta u^{\prime} \\
\Delta u^{\prime \prime}
\end{array}\right)=0
$$

or simply as

$$
a \Delta u+b \Delta u^{\prime}+c \Delta u^{\prime \prime}=0 .
$$

So the geometry of three uncalibrated affine 1D views is completely characterised by this linear constraint represented by the homogeneous 3 -vector $(a, b, c)^{T}$ which has only 2 d.o.f. With at least 3 point correspondences in three views, 2 relative points plus the reference point, the vector $(a, b, c)^{T}$ could be estimated linearly.
The above three-view linear constraint directly encodes the relative motion parameters. For reconstruction, similar to factorization method [26] for 2D affine cameras, we could proceed the same way by stacking $p$ points in $n$ images to create the measurement matrix as

$$
\left(\begin{array}{ccc}
\Delta u_{1} & \ldots & \Delta u_{p} \\
\Delta u_{1}^{\prime} & \ldots & \Delta u_{p}^{\prime} \\
\ldots & \ldots & \ldots \\
\Delta u_{1}^{(n)} & \ldots & \Delta u_{p}^{(n)}
\end{array}\right)=\left(\begin{array}{c}
\mathbf{m}^{T} \\
\ldots \\
\mathbf{m}^{(n)^{T}}
\end{array}\right)\left(\begin{array}{lll}
\Delta \mathbf{x}_{1} & \ldots & \Delta \mathbf{x}_{p}
\end{array}\right)
$$

or in compact form as $\mathbf{U}_{n \times p}=\mathbf{M}_{n \times 2} \mathbf{S}_{2 \times p}$. The rank of the $n \times p$ measurement matrix can not exceed 2. By keeping the two largest singular values and zeroing all others of the measurement matrix, we obtain the best ranktwo camera matrices $\mathbf{m}_{i}$ and point reconstruction $\Delta \mathbf{x}_{i}$.

As for any non singular $2 \times 2$ matrix $\mathbf{A}$ representing a plane affine transformation, $\mathbf{U}=\mathbf{M S}=(\mathbf{M A})\left(\mathbf{A}^{-1} \mathbf{S}\right)=$ $\mathbf{M}^{\prime} \mathbf{S}^{\prime}$. The camera matrix and shape are still affine. We need to look for a $\mathbf{A}$ such that $\mathbf{m}_{i} \mathbf{A}=c \mathbf{R}_{1 \times 2}$ where $c$ is an independent scaling factor that each 1D affine camera may have. So that the metric constraint for euclidean reconstruction assuming constant scaling factor is

$$
\mathbf{m}_{i} \mathbf{A} \mathbf{A}^{T} \mathbf{m}_{i}^{T}=1 .
$$

This reconstruction might be sufficient if not it can be served as an initial solution for a nonlinear optimisation. The factorisation method requires all points in all images. For missing points, they can be handled using the linear three-view constraint developed in the previous section.

\subsection{Reconstruction from calibrated 1D panorama projective images}

The 1D affine camera nicely describes the geometry for the original images within the same concentric mosaic. For recovering relative orientation and depth from concentric mosaics at different positions for large-scale environement modelling, each concentric mosaic is assimilated into a calibrated 1D panorama projective camera. We describe a complete reconstruction method from 3 panorama 1d projective cameras which assembles the recent results in $[3,4,20,9]$ for $1 \mathrm{D}$ cameras.

- Computing uncalibrated 1D trifocal tensor The geometry of three 1D images is completely characterised by the $1 \mathrm{D}$ trifocal tensor $\mathbf{T}_{i j k}$. It minimally parametrizes the three uncalibrated images and it can be estimated linearly with at least 7 point correspondences.

- Solving a cubic equation for the internal parameters The 1D camera could be self-calibrated for constant calibration parameters via 1D trifocal tensor [9]. The knowledge of the internal parameters 
pair of complex conjugate points can be uniquely determined by solving the cubic equation: $T_{111} x^{3}+$ $\left(T_{211}+T_{112}+T_{121}\right) x^{2}+\left(T_{212}+T_{221}+T_{122}\right) x+$ $T_{222}=0$.. The real part of the ratio of the projective coordinates of the image of the circular point $\mathbf{i}$ is the position of the principal point $u_{0}$ and the imaginary part is the focal length $\alpha$.

- Computing calibrated 1D trifocal tensor The internal parameters of the camera can either be selfcalibrated as described in the previous section or given by off-line calibration, then we come to the case of calibrated 1D camera. To handle calibrated geometry properly, the image coordinates could be first normalised by applying $\mathbf{K}^{-1}\left(u_{i}, 1\right)^{T}$ to get $\left(x_{i}, 1\right)^{T}$. To see what happens for the calibrated trifocal tensor, it suffices to notice that knowing the internal parameters of a 1D camera is equivalent to knowning two points, the pair of circular points! Substituting the circular points $( \pm i, 1)$ into the trilinear constraint gives the two following scalar constraints [4] $T_{122}+T_{212}+T_{221}-T_{111}=0$ and $T_{112}+T_{121}+T_{211}-T_{222}=0$.

The 1D trifocal tensor can now be linearly reestimated by taking into account of these constraints. Substituting $T_{111}$ and $T_{222}$ back into the original trilinear constraint equation gives constrained trilinear constraint.

- Recovering calibrated projection matrix For reconstruction, we need to convert the tensor compoenent into camera projection matrices. Each projection matrix can be parameterized by

$$
\left(\begin{array}{ccc}
c & -s & t_{x} \\
s & c & t_{y}
\end{array}\right)
$$

Actually there are 5 non-homogeneous tensor components, and 5 d.o.f. for P-matrices, $\theta$ and $\theta^{\prime}, \mathbf{t}$ and $\mathbf{t}^{\prime}$ up to a scale. This can be solved algebraically, but up to a two-way ambiguity $[4,20]$.

- Reconstructing 2D point coordinates Each 2D point can be reconstructed by solving linear equations provided by $\lambda(u, 1)^{T}=\mathbf{M}_{2 \times 3}(x, y, 1)^{T}$.

- Nonlinear optimisation Finally, the reconstruction can be improved by a nonlinear optimisation method.

\subsection{D reconstruction from calibrated 1D pro- jective images of a circular motion}

Though the affine approximation is often sufficient for images from the same CM as described in Section 4.2, we may still apply a full $1 \mathrm{D}$ projective camera model. The same reconstruction algorithm as described in the previous subsection is still valid, but the images from the from the same CM has stronger motion constraint: a circular motion [10]. The calibrated $2 \times 3$ projection matrices for a triplet of views can be parameterized as

$$
(\mathbf{R}(\theta), \mathbf{t}),\left(\mathbf{R}\left(\theta^{\prime}\right), \mathbf{t}\right), \text { and }\left(\mathbf{R}\left(\theta^{\prime \prime}\right), \mathbf{t}\right) .
$$

The associated trifocal tensor has also two additional constraints than the calibrated 1D trifocal tensor. One is that $T_{222}=0$ if we choose $t_{y}=0$ without loss of generality. The other has more complicated expression. This particular parametrisation also suggests a more efficient bundle-like nonlinear optimisation.

\section{Experimental results}

Experiments on analyzing concentric mosaic data by $1 \mathrm{D}$ cameras have been carried out. In this section, we show some preliminary results based on tracking results of points of interest from triplets of original images captured by the concentric mosaic set-up in our lab.

KIDS sequence For the KIDS triplet shown in 2, there are 159 and 107 match candidates in the first and second pairs. We obtain 89 final match triplets. The 3D affine reconstruction using standard $2 \mathrm{D}$ factorization method is shown in Figure 3. The horizontal plane is referenced by coordinates $(x, z)$, so the $z$-coordinate gives the depth and the $y$-coordinate the height. The 2D affine and Euclidean reconstruction using our 1D factorisation method is shown in Figure 4.

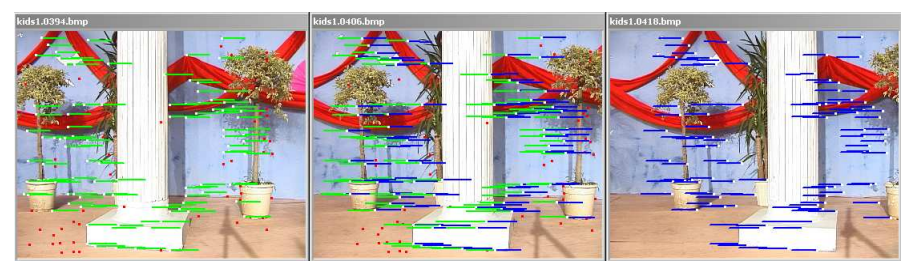

\section{Figure 2. Point correspondences in a triplet from KIDS sequence.}

In Figure 5, two columns and the backgroup wall are drawn over the reconstructed plane to illustrate the reconstruction quality.

TOY sequence For the TOY triplet illustrated in Figure 6 , there are 151 and 126 match candidates in the first and second pairs. 76 final corresponding triplets are obtained. The 3D affine reconstruction using standard 2D factorisation method is shown in Figure 7. The 2D affine and Euclidean reconstruction using our 1D factorisation method are shown in Figure 8. We can notice the superior reconstruction by $1 \mathrm{D}$ factorisation method over the $2 \mathrm{D}$ factorisation.

The final 3D VRML model shown in Figures 10 and 9 are reconstructed from re-sampled dense matching by the 


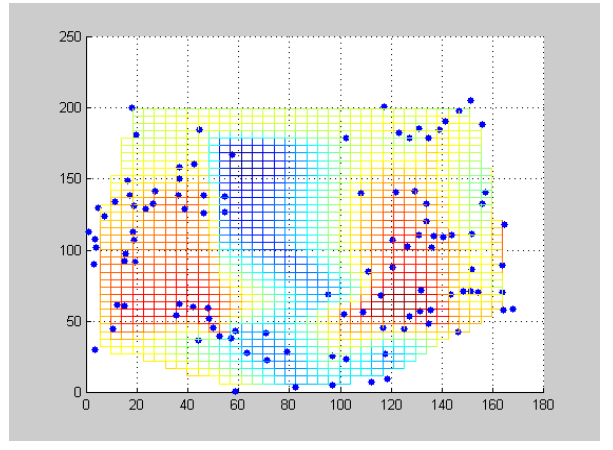

Figure 3. 3D Affine reconstruction by 2D factorisation for KIDS sequence: projection onto $(x, z)$ plane.

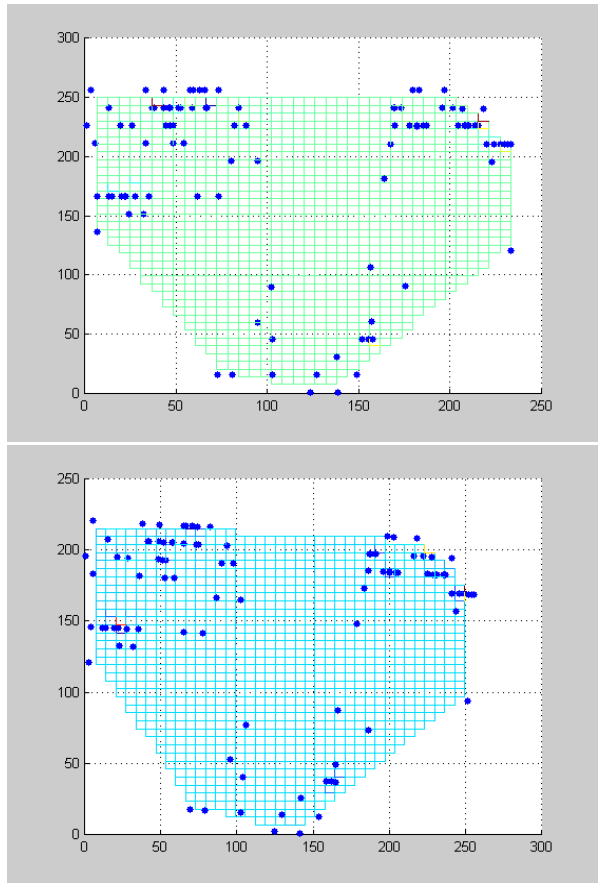

Figure 4. 2D Affine and Euclidean reconstruction by 1D factorisation for KIDS sequence.

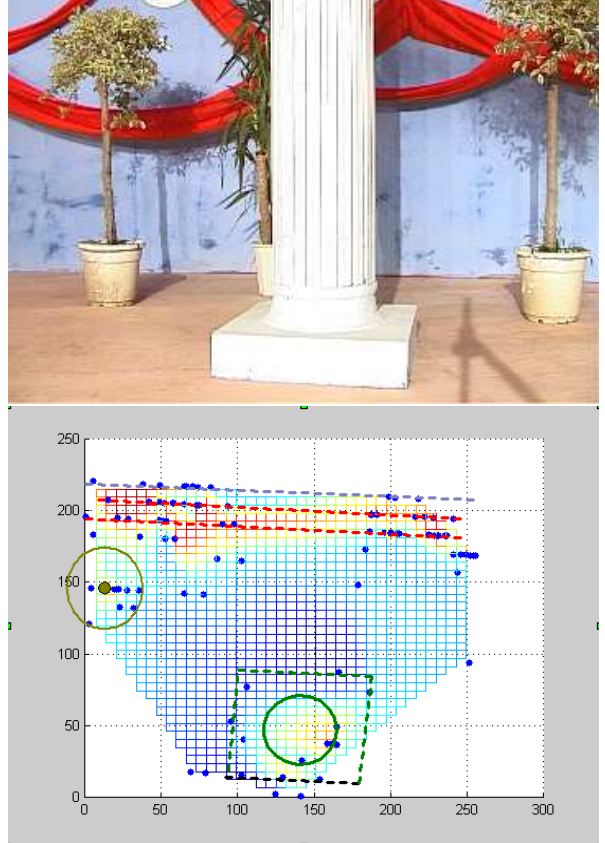

Figure 5. One original image and the reconstructed plane by merging two triplets of KIDS sequence with manual drawing for illustration.

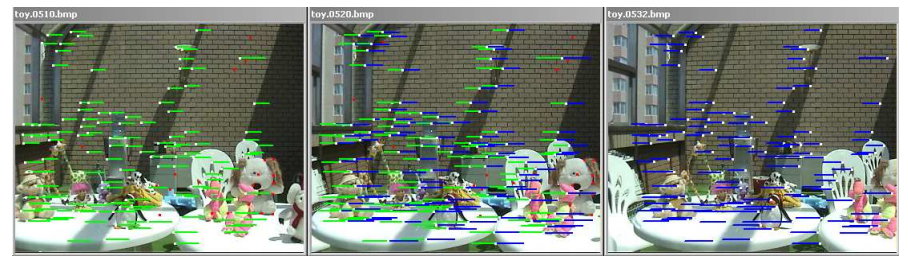

Figure 6. Point correspondences in a triplet from TOY sequence.

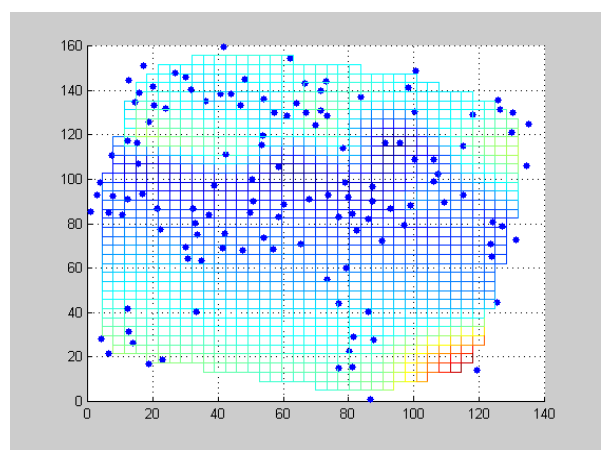

Figure 7. 3D Reconstruction by 2D factorisation method for TOY triplet. 
Research Beijing for support and B. Triggs and P. Sturm for fruitful discussions. The work has also been partly supported by EU IST Project EVENTS.

\section{References}

[1] M. Armstrong, A. Zisserman, and R. Hartley. Selfcalibration from image triplets. ECCV'96.

[2] M.N. Armstrong. Self-Calibration from Image Sequences. $\mathrm{PhD}$ thesis, University of Oxford, 1996.

[3] K. Åström. Invariance Methods for Points, Curves and Surfaces in Computational Vision. PhD thesis, Lund University, 1996.

[4] K. Åström and M. Oskarsson. Solutions and ambiguities of the structure and motion problem for $1 \mathrm{~d}$ retinal vision. JMIV, 2000.

[5] S. Avidan and A. Shashua. Novel view synthesis in tensor space. CVPR'97.

[6] P.A. Beardsley and A. Zisserman. Affine calibration of mobile vehicles. Europe-China Workshop on Geometrical Modelling and Invariants for Computer Vision, 1995.

[7] S.E. Chen. Quicktime VR - an image-based approach to virtual environment navigation. SIGGRAPH'95.

[8] O. Faugeras and B. Mourrain. About the correspondences of points between $n$ images. Workshop on Representation of Visual Scenes, 1995.

[9] O. Faugeras, L. Quan, and P. Sturm. Self-calibration of a 1d projective camera and its application to the self-calibration of a $2 \mathrm{~d}$ projective camera. ECCV'98.

[10] A.W. Fitzgibbon, G. Cross, and A. Zisserman. Automatic 3D Model Construction for Turn-Table Sequences. SMILE'98.

[11] S.J. Gortler, R. Grzeszczuk, R. Szeliski, and M. Cohen. The lumigraph. SIGGRAPH' 96.

[12] R.I. Hartley. A linear method for reconstruction from lines and points. ICCV'95.

[13] E. Izquierdo and S. Kruse. Image analysis for 3d modeling, rendering and virtual view generation. CVIU, 1998.

[14] S. Laveau and O. Faugeras. 3D scene representation as a collection of images and fundamental matrices. TRINRIA, 1994.

[15] M. Levoy and P. Hanrahan. Light field rendering. SIGGRAPH'96.

[16] L. McMillan and G. Bishop. Plenoptic modeling: An image-based rendering system. SIGGRAPH'95.

[17] J.L. Mundy and A. Zisserman. Projective geometry for machine vision. Geometric Invariance in Computer Vision, 1992.

[18] P.J. Narayanan, P.W. Rander, and T. Kanade. Constructing virtual worlds using dense stereo. ECCV'98.

[19] S. Peleg and J. Herman. Panoramic mosaics by manifold projection. CVPR'97.
[21] P.W. Rander, P.J. Narayanan, and T. Kanade. Recovery of dynamic scene structure from multiple image sequence. Int'l Conf on Multisensor Fusion and Integration for Intelligent Systems, 1996.

[22] A. Shashua. Algebraic functions for recognition. PAMI'95.

[23] H.Y. Shum and L.W. He. Rendering with concentric mosaics. SIGGRAPH'99.

[24] M. Spetsakis and J. Aloimonos. A unified theory of structure from motion. DARPA Image Understanding Workshop, 1990.

[25] R. Szeliski and H.-Y. Shum. Creating full view panoramic image mosaics and environment maps. SIGGRAPH'97.

[26] C. Tomasi and T. Kanade. Factoring image sequences into shape and motion. IJCV'92.

[27] B. Triggs. Matching constraints and the joint image. ICCV'95.

[28] B. Triggs. Plane + parallax, tensors and factorization. ECCV'2000.

[29] C. Wiles and M. Brady. Ground plane motion camera models. ECCV'96. 